\title{
IMMEDIATE HEALTH CARE MONITORING SYSTEM USING IoT Devices
}

\author{
Mr. Ajay Rastogi \\ Assistant professor, \\ College of Computing Sciences \& Information Technology, \\ Teertanker Mahaveer University, Moradabad-244001
}

\begin{abstract}
Internet of things can be used very effectively in the healthcare. It can be a real time solution for the patient. When a patient is suffering from any disease he/she is not capable to take care of him/herself. They need some extra care and if the disease is very critical then it is very tedious to get every second to a doctor. This is a major problem. And the caretaker could not understand what to do in such situation. IoT can be a one of the best solution for this problem. One can easily set some sensors on the patents body and doctor can easily got the details. if require he can change the medicines and advice at a very emergency time when there is no need to move the patient outside the home.
\end{abstract}

Key words: Internet of things (IoT)

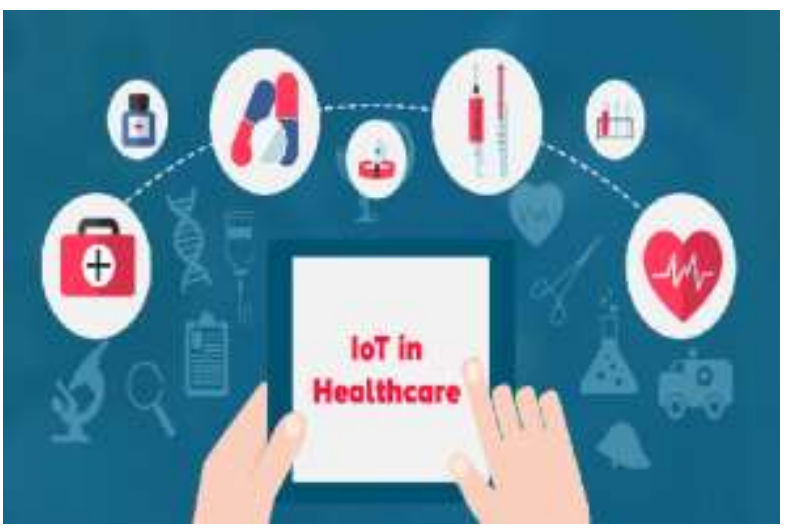

I. INTRODUCTION

In the present scenario people are too much busy and many of their work have to be done by different machines. In this scenario different machines are helping humans to get relax and sit back. These machines are capable to communicate with other machines as well as humans. And all of this is made possible due to the integration of electronics, mechanic with computing capability and the rise of internet of things(IoT). IoT is not a very old technology. It is very young technology and facts are shown that these devices are the very much capable to do many things, many important task of human life. By IoT we can also get our patient to be monitored by the doctor any time whether he is present or not.

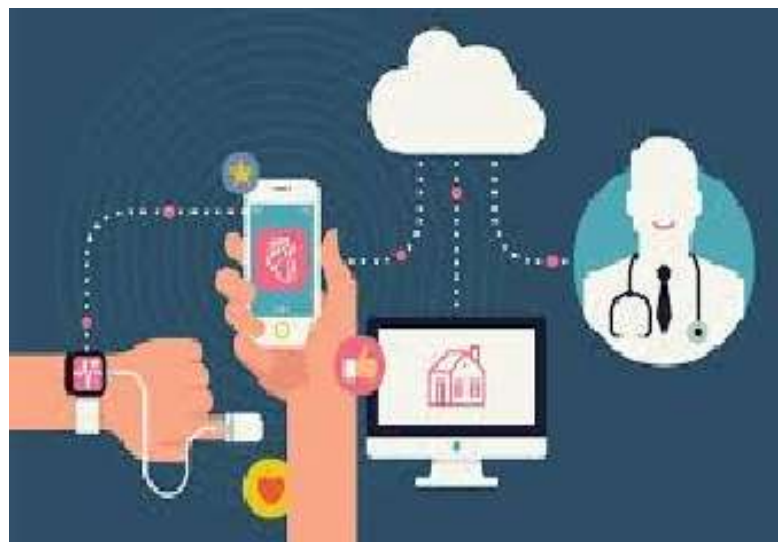

Internet of things(IoT) have change the scenario of today. There are many different problem is solving by the use of IoT. Here we are discussing the special yet important task of a human life using the IoT devices i.e. Healthcare. Many times there is a situation when a patient have a typical pain in his chest in the night and at his home. And no doctor is available to there. Today one can make a video call to doctor to see but what if doctor want to check him up, at that time sensors are comes into the picture. A known person put the sensors on the body of the patient and send immediate data to the doctors. A doctor can receive the same and check the criticality and diagnose as necessary. So with the IoT devices one can save a life of a person by getting accurate advice from the doctor without moving towards hospitals.

Today, a commonly accepted definition for IoT is a dynamic global network infrastructure with selfconfiguring capabilities based on standard and interoperable communication protocols where 
International Journal of Engineering Applied Sciences and Technology, 2019

Vol. 4, Issue 3, ISSN No. 2455-2143, Pages 286-288

Published Online July 2019 in IJEAST (http://www.ijeast.com)

physical and virtual 'Things' have identities, physical attributes, and virtual personalities and use intelligent interfaces, and are seamlessly integrated into the information network "R. van Kranenburg (2007)".

\begin{tabular}{l|l}
\multicolumn{2}{c}{ TABLEI } \\
\hline Layers & Description \\
\hline Sensing layer & $\begin{array}{l}\text { This layer is integrated with existing } \\
\text { hardware (RFID, sensors, actuators, } \\
\text { ete) to sense/control the physieal } \\
\text { world and acquire data }\end{array}$ \\
\hline Networking layer & $\begin{array}{l}\text { This layer provides basic networking } \\
\text { support and data transfer over } \\
\text { wireless or wired network }\end{array}$ \\
\hline Service layer & $\begin{array}{l}\text { This layer creates and manages } \\
\text { services. It provides services to } \\
\text { satisfy user needs }\end{array}$ \\
\hline Interface layer & $\begin{array}{l}\text { This layer provides interaction } \\
\text { methods to users and other } \\
\text { applications }\end{array}$
\end{tabular}

Devices in the form of wearables like fitness bands and other wirelessly connected devices like blood pressure and heart rate monitoring cuffs, glucometer etc. give patients access to personalized attention. These devices can be tuned to remind calorie count, exercise check, appointments, blood pressure variations and much more.

IoT has changed people's lives, especially elderly patients, by enabling constant tracking of health conditions. This has a major impact on people living alone and their families. On any disturbance or changes in the routine activities of a person, alert mechanism sends signals to family members and concerned health providers.

The four stages of IoT solutions $\mathrm{Li} \mathrm{Da} \mathrm{Xu}, \mathrm{Wu} \mathrm{He}$, and Shancang Li. (2014, Nov.)

As in the following figure one can easily understand the process of implementation of IoT in healthcare.

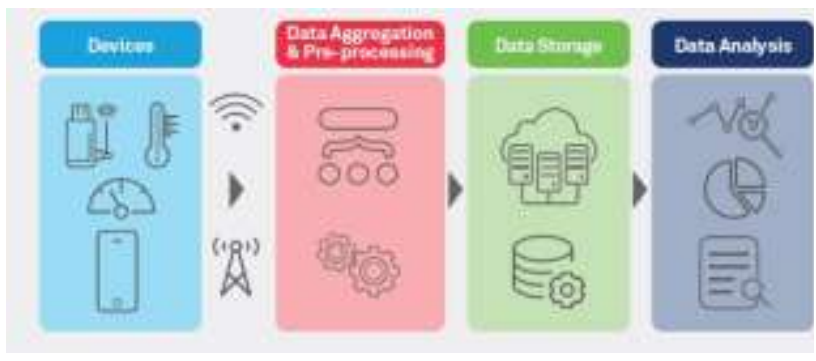

There are four Component of IoT Healthcare-

- Sensing- consists of all sensors, RFID, wireless sensor network(WSN) etc., e.g. Google glass, fitbit sensors.
- Aggregating- Aggregation of different sensor data is also. It consist different type of aggregators.

- Processing-Here all the data have to be processed.

- Cloud-the main component by which one can access the data.

The major advantages of IoT in healthcare include:

- Cost Reduction: IoT enables patient monitoring in real time, thus significantly cutting down unnecessary visits to doctors, hospital stays and re-admissions

- Improved Treatment: It enables physicians to make evidence-based informed decisions and brings absolute transparency

- Faster Disease Diagnosis: Continuous patient monitoring and real time data helps in diagnosing diseases at an early stage or even before the disease develops based on symptoms

- Proactive Treatment: Continuous health monitoring opens the doors for providing proactive medical treatment

- Drugs and Equipment Management: Management of drugs and medical equipment is a major challenge in a healthcare industry. Through connected devices, these are managed and utilized efficiently with reduced costs

- Error Reduction: Data generated through IoT devices not only help in effective decision making but also ensure smooth healthcare operations with reduced errors, waste and system costs

\section{FUTURE WORK}

We can develop several sensors that can sense the patient data accurately. There are some limited type of sensors. There is a need to develop different type of sensors to get perhaps all types of statistics from a patient that is necessary. For example we are having sensors for blood pressure and pulse measurement and also we have some sensors that are used with ECG(Electro-Cardiogram) but we require more and more to integrated with the IoT devices such as sugar checker sensors. Some sensors need to be developed for some common blood test, etc. 


\section{International Journal of Engineering Applied Sciences and Technology, 2019}

Vol. 4, Issue 3, ISSN No. 2455-2143, Pages 286-288

Published Online July 2019 in IJEAST (http://www.ijeast.com)

\section{CONCLUSION}

As we can see that with the help of internet of things (IoT) devices we can easily consult with doctor in emergency time. Or we can regularly keep in touch with doctor. A doctor can see and check the patient statistics anytime according o his convenience.

\section{REFERENCES}

[1] Li $\mathrm{Da} \mathrm{Xu}, \mathrm{Wu} \mathrm{He}$, and Shancang Li. (2014, Nov.). Internet of things. in Industries: A Survey Article in IEEE Transactions on Industrial Informatics

[2] R. van Kranenburg (2007). , The Internet of Things: A Critique of Ambient Technology and the All-Seeing Network of RFID. Amsterdam, The Netherlands: Institute of Network Cultures, 2007.

[3] Dr. Rajashekhar Karjagi Head - Analytics Solutions, Wipro, Manish Jindal Manager Analytics Solutions, Wipro, What can do IoT for healthcare? available online https://www.wipro.com/business-process/what-caniot-do-for-healthcare- 1

[4] B. Karakostas(2013), "A DNS architecture for the internet of things: A case study in transport logistics," Procedia Comput. Sci., vol. 19, pp. 594-601, 2013. 